Science \& Society

Series: Seminal Neuroscience Papers 1978-2017

\title{
The Rough Seas of Cortical Cartography
}

Semir Zeki.

s.zeki@ucl.ac.uk

University College London, London, UK

Correspondence:

Efforts to parcellate the cortex into areas based on fine-scale anatomical signatures (e.g. cytoarchitectonics) date back to the early years of the last century. In the ensuing decades, rapidly growing knowledge about of cortical connections encouraged neurobiologists to search for connectivity-based principles underlying the organization of the cerebral cortex. Using such an approach, the 1991 paper by Felleman and Van Essen presented a connectivity, in their 1991 paper, a connectivity-based hierarchical principle dictating the relationship between cortical areas. This helped invigorate debates on the principles underlying cortical organization, including searches for alternative, or complementary, principles.

Keywords: cortical organization; cortical hierarchies; cortical connections

Cortical anatomy has dominated attempts to give an all-embracing account of how the cerebral cortex is organized. Myeloarchitectonics, cytoarchitectonics, myelogenesis, and multimodal magnetic resonance imaging (MRI) are some of the approaches that have been used to try and characterize cortical architecture. These techniques have, between them, divided the cerebral cortex into variable numbers of areas, some approaching as many as 200. An implicit assumption behind these analyses has often been that anatomically distinct subdivisions of the cortex are also functionally distinct. With this as a basis, cortical cartographers did not have to pay much attention to the question of what functions could be assigned to their subdivisions. FAlthough none of the resulting schemas has given a satisfactory, all-embracing account of the anatomical principle underlying cortical organization, something of value has remained from each of them. From an an historical perspective, one could argue that these schemas, although using different means, represent a search for an elusive principle that governs the organization of the cerebral cortex, $\frac{1}{2}$ which none was able to fully establish. ButBut However, the debates that these schemas generated touched on important questions regarding the organization of the cerebral cortex, which remain as relevant today as when first raised.

Broadly speaking, two general goals motivate the anatomy-based attempts to characterize cortical organization. The first is to divide the cortex into a set of anatomically-_defined areas. In that regard, the most frequently cited map so far has been Korbinian Brodmann's, which divided the cerebral cortex into aboutpproximately about 50 areas. A second aim, once a list of areas is 
defined, has been to assemble these into larger structures, or networks, based on the anatomical projection patterns among the areas.

The primary and the most commonly referred-to example of a study driven by this second goal is the 1991 paper by Felleman and Van Essen [1] (hereinafter the F\&VE paper). Its influence is reflected by the sheer number of its citations, the fairly wide adoption of some of its central theses, particularly that of hierarchical organization, and the regular use of its summary diagram in public lectures to illustrate the complexities of the brain. At its core, the paper is a meta-analysis of the laminar organization of connections between 32 cortical areas, mostly ones involved in some way or another in visual functions. The authors used the results of cortical connectivity studies to give a new definition of hierarchy in the cerebral, and especially the visual, cortex. Their proposed schema gives each visual area a determined position, derived from this pattern of connections, within an overall "single hierarchy" single hierarchy".

The theory embraced by the paper is based largely on the organization of the visual cortex, and adopts a principle first enunciated by Rockland and Pandya [2], to the effect that forward connections (i.e., those leading away from the primary visual cortex, area V1) could be distinguished from backward connections on the basis of the laminar organization of their terminations. Specifically, the forward connections emanate from the superficial layers and terminate predominantly in layer 4 of the recipient area, whereas the reciprocal, reverse, connections originate in both superficial and deep layers and terminate outside layer 4. This apparent regularity was subject to certain variations and ambiguities, but such departures from the general principle of a "strict hierarchy" strict hierarchy" did not affect materially the overall schema, or so it was assumed.

Altogether, the authors documented 305 identified pathways in the anatomical literature, with many of these pathways (242) representing reciprocal connections between areas (121 pairs of connections). These numbers reflect, in part, the rapidly growing bank of data on primate cortical anatomy induring the 1970s and 1980s. To effectively process this information, the authors took an approach that may seem mundane nowadays but was relatively prescient at the time. First, they generated a computerized database of the connections of the brain areas being examined. Second, given the complexity of the resulting visualizations, they actualized the schema on a graphics-drawing--program that allowed users to inspect the diagram in a flexible manner, for instance by switching on or off each of the areas and its corresponding connections.

The F\&VE paper was especially successful in projecting a particular view of the cerebral cortex in general, and of the visual cortex in particular, namely that cortical areas can be allocated a given, unique, position in an overall "single hierarchy" "single hierarchy", based on the identification of forward, backward, and lateral patterns of anatomical connections that they establish with each other. In the visual cortex, the lowest area was taken to be V1 f[which receives 
visual signals from the retina through the lateral geniculate nucleus (LGN) $]$ and feeds the further visual areas in the prestriate cortex surrounding it, and beyond. This, in turn, led to the general belief that there are "tiers" "tiers' of areas, broadly assignable to the "low" "middle" or "high"'low', "middle', or 'high' level categories, a view especially, and uncritically, espoused by those working on the visual brain's form system (see [3] for a reviewform system of the visual brain (reviewed in [3]). A general supposition, which seemappeared to support the emphasized hierarchical strategy, was that the functional properties of cells become more complex in successive areas within a hierarchy, for which the most impressive evidence at the time (outside V1) was the increase in the receptive field size of cells in successive areas.

As has become clear since, and as discussed below, the attempt to channel all cortical connections into a single hierarchical schema faces varioussignificant difficulties. The central and most widely adopted conclusion of the F\&VE paper, that regarding hierarchies, was disavowed by Hegdé and Felleman in a review published 16 years later [4], where they wrote, “- visual processing neither is hierarchical nor parallels the anatomical hierarchy" and, that " and, that 'while the sustaining strength of the notion of hierarchical processing may be that it is rather simple, its fatal flaw is that it is overly simplistic"' [4]. The contrast between this perspective and the one of the F\&VE paper, encapsulates, in a way, the novel questions about cortical organization that have arisen during the time between the publication of these two papers.

Below are some of the difficulties around the 'single-hierarchy' view of visual processing. The first difficulty relates to the starting point of the proposed hierarchy, namely V1. Positioning V1 at the entry point of the cortical visual hierarchy echoes the age-old supposition that $\mathrm{V} 1$ is “"the [sole] -entering place of the visual radiation into the organ of psyche" [8]. In fact' [5]. In fact, it had been established relatively early on that the LGN [5,66,7] and the thalamic pulvinar nucleus [7 $\underline{7}$ ] project directly to the specialized visual areas of prestriate cortex, thus bypassing V1. These pathways call into question the very foundation of a visual cortical visual hierarchy - namely V1's role, namely the role of V1 in it; but not many took much notice of that then (and not many do so now). The existence of the anatomical subcortical-_prestriate pathways relates to another important observation, which only became known sinceafter the publication of the F\&VE paper, namely the arrival times of signals in the visual brain. Specifically, visual signals arrive in the prestriate areas with the same, or even shorter, latencies than they arrive in V1, depending upon stimulus and task (3)[3]. Moreover, the direct thalamic input into prestriate visual areas such as V5 (MT) is potent enough to sustain a conscious, if crude, experience of directional visual motion (9)[9]. Another difficulty with the 'single' hierarchical hierarchical' model relates to the prediction from the hierarchical scheme that "higher"'higher" areas have more complex functions than "lower"'lower' ones. This 
prediction has not been universally borne out by physiological (10) or perceptuat experiments[10] or perceptual (masking) experiments (11). Finally, there are challenges also[11]. Finally, there are also challenges around the notion of grouping areas into lateral hierarchical "tiers" "tiers'. For instance, many psychophysical pairing experiments show that, at very early perceptual stages, we do not see all visual attributes simultaneously, even if the percepts are the result of activity in areas which whichthatwhich are deemed to belong to the same hierarchical "tier" "tier' of the F\&VE schema [12]. In summary, the perceptual hierarchy cannot be predicted from the anatomical hierarchy; the temporal hierarchy in the arrival of signals in V1 and the visual areas surrounding it [13] cannot be predicted from the classic anatomy espoused in the F \& $\underline{\&}$ VE paper and in most other papers too; and the sequence of arrival of signals in the cortex does not predict the perceptual hierarchy, at least within very brief time frames $(<\leq 150 \mathrm{~ms})$. Hence, the hierarchy traceable to the pattern of laminar terminations seemappears to be only one among various hierarchies that operate in the brain.

Much more is known today about cortical organization than was known three decades ago. The F\&\& \&E paper eould-would have aged better if facts known now were known then or facts known then had been more prominently established. Nevertheless, again like likesimilar to-other cartographic studies, something important and lasting remains from it, namely the anatomical relationship established between areas in terms of their connections. There is also no doubt of its historical interest, because it has led so many to espouse a view that has, in its simplicity and in the words of one of its authors, "a fatal flaw". Ultimately, inter 'a fatal flaw'. Ultimately, interareal relationships as inferred from anatomical connectivity isare only one of the organizing-_principles that operate in the brain; there are others, and how they can all be weaved into a single principle that governs how the cortex functions remains as elusive as it has always been.

\section{Uneited references $[9$ 11, 14].References}

1. D.J. Felleman and D.C. Van Essen, Distributed hierarchical processing in the primate cerebral cortex, Cereb, Cortex 1, 1991, 1-47.

2. K.S. Rockland and D.N. Pandya, Laminar origins and terminations of cortical connections in the occipital lobe, Brain Res-. 179, 1979, 3-20.

3. S. Zeki, Multiple asynchronous stimulus- and task-dependent hierarchies (STDH) within the visual brain's parallel processing systems, Eur. J. Neurosci_. 44, 2017, 2515-2527.

4. J. Hegdé and D.J. Felleman, Re-appraising the functional implications of the primate visual anatomical hierarchy, Neuroscientist 13, 2007, 416-421.

5. 8Flechsig, P. (1905).Flechsig, P. (1905) Gehirnphysiologie und Willenstheorienpp 73 89i. In Some Papers on the Cerebral Cortex. (von Bonin, G. et al., trans), pp. 73-89 
6. M. Yukie and E. Iwai, Direct projection from the lateral geniculate nucleus to the prestriate cortex in macaque monkeys, J. Comp. Neurol. 201, 1981, 81-97.

67. W. Fries, The projection from the lateral geniculate nucleus to the prestriate cortex of the macaque monkey, Proc. R. Soc. Lond. B 213, 1981, 73-80.

72. B.G. Cragg, The topography of the afferent projections in the circumstriate visual cortex of the monkey studied by the Nauta method, Vis. Res. 9, 1969, 733-747.

(trans G. ven Benin, Springfield, I, CC Thomas.9 S. Zeki and D.H. fFfytche, The Riddoch Syndrome: insights into the neurobiology of conscious vision, Brain 121, 1998, 25-45.

10. J. Hegdé and D.C. Van Essen, A comparative study of shape representation in macaque visual area V2 and V4, Cereb. Cortex 17, 2007, 1100-1116.

11. Y.T. Lo and S. Zeki, Masking reveals parallel form systems in the visual brain, Front. Hum. Neurosci8201810810.3389/fnhum.2014.00567. 8, 2014, 108.

12. K. Moutoussis and S. Zeki, Functional segregation and temporal hierarchy of the visual perceptive systems, Proc. R. Soc. Lond. B 264, 1997, 1407-1414.

13. Y. Shigihara and S. Zeki, Parallelism in the brain's visual form system, Eur. J. Neurosci. 38, 2013, $3712-3720$. 